\title{
Qualità della vita nei pazienti con microcarcinoma papillare della tiroide in funzione del trattamento: tiroidectomia totale con o senza terapia radiometabolica ablativa
}

\author{
Antonio Matrone $^{1} \cdot$ Rossella Elisei $^{1}$
}

Accettato: 6 agosto 2020 / Pubblicato online: 30 ottobre 2020

(c) The Author(s) 2020

\section{Commento a:}

Quality of life in patients with papillary thyroid microcarcinoma according to treatment: total thyroidectomy with or without radioactive iodine ablation.

\section{J. Ahn, M.J. Jeon, E. Song, T.Y. Kim, W.B. Kim,} Y.K. Shong, W.G. Kim.

Endocrinol Metab (2020) 35:115-121

Gli autori di questo lavoro hanno voluto esplorare l'impatto sulla qualità della vita (QoL) dell'aggiunta di un trattamento radiometabolico (RAI) alla tiroidectomia totale nei pazienti affetti da carcinoma differenziato della tiroide (DTC), dato che nei pazienti a basso rischio di recidiva e, in particolare, nei microcarcinomi papillari (mPTC), come confermato nelle ultime linee guida di consenso [1], l'utilità della RAI è altamente controversa. A tale scopo gli autori hanno valutato prospetticamente 289 pazienti con DTC trattati fra giugno 2016 e ottobre 2017. Alcuni soggetti con determinate caratteristiche (diagnosi pregressa o attuale di disturbi psichiatrici, patologie reumatologiche, altri cancri, ipertensione non controllata, trattamento insulinico per diabete) sono stati esclusi.

Sono stati valutati età, sesso, stato coniugale, livello di educazione, impiego e stato socioeconomico. Anche dati di laboratorio, inclusa la valutazione del valore di TSH al momento della compilazione del questionario, sono stati inclusi e valutati nello studio.

I pazienti con $\mathrm{mPTC}$ che eseguivano RAI venivano trattati con $30 \mathrm{mCi}$ eccetto quelli che presentavano metastasi lin-

R. Elisei

rossella.elisei@med.unipi.it

1 Dipartimento di Medicina Clinica e Sperimentale, Unità di Endocrinologia 1, Azienda Ospedaliera Pisana, Università di Pisa, Pisa, Italia fonodali del comparto centrale (N1a), che venivano trattati con $80 \mathrm{mCi}$.

Per la valutazione della QoL tutti i pazienti completavano 3 questionari: il 12-item Short-Form health survey (SF-12), il Thyroid Cancer-Specific Quality of Life (THYCA-QoL) e il Fear of Progression (FoP).

Il SF-12 è la forma breve del SF-36, che consta di 12 domande che danno un profilo dello stato di salute includendo diversi item: la salute fisica, limitazioni dovute alla salute fisica, limitazioni dovute a problemi emozionali, salute generale, vitalità, dolore corporeo, funzionamento sociale e salute mentale. I risultati sono sintetizzati in uno score di salute fisica e mentale che va da 0 a 100 . Più basso è lo score, più lo stato di salute è considerato peggiore. Il THYCA-QoL contiene invece 24 item che valutano i sintomi relativi al carcinoma tiroideo in sé o in seguito al suo trattamento. Ogni item contiene un punteggio da 1 (poco) a 4 (molto) che viene convertito, poi, in due score. Più basso è lo score, minore è il disturbo dovuto ai sintomi. FoP valuta invece l'ansia e la paura di progressione di malattia ed è basato su 43 item che valutano le reazioni affettive, il rapporto con il partner e la famiglia, il lavoro, la perdita di autonomia e il far fronte all'ansia. Uno score più alto indica più ansia di progressione di malattia per tutte le categorie, tranne per l'ultima, dove uno score più elevato indica una migliore capacità di far fronte all'ansia.

Dei 289 pazienti analizzati, 107 avevano eseguito solo chirurgia (gruppo TTx) e 182 avevano eseguito chirurgia e RAI (gruppo TTx+RAI). Nessuno dei pazienti del gruppo TTx aveva metastasi linfonodali; di contro, ben 106 (58\%) pazienti del gruppo TTx+RAI avevano N1a. Nel gruppo TT+RAI, 72 (40\%) pazienti erano stati trattati con $30 \mathrm{mCi}$, mentre $110(60 \%)$ con $80 \mathrm{mCi}$ per la presenza di minima estensione extratiroidea o N1a. Solo 40 pazienti (22\%) venivano preparati per la RAI con TSH umano ricombinante 
(rhTSH), tutti gli altri con ipotiroidismo. Al momento della compilazione del questionario (mediana 3,5 anni dopo il trattamento iniziale, senza differenze significative fra i due gruppi), nessuno aveva malattia strutturale residua.

Il gruppo TT+RAI era più frequentemente sposato $(p=$ $0,031)$ e presentava valori più bassi di TSH $(p<0,001)$. Vista questa differenza, i gruppi sono stati abbinati per valori di TSH, portando il numero dei pazienti a 100 per ogni gruppo. Dopo l'abbinamento i due gruppi rimanevano uguali per caratteristiche basali. Nessuno al data lock dello studio (novembre 2019) presentava una recidiva di malattia.

Nell'analisi di SF-12, i pazienti TTx+RAI presentavano punteggi significativamente più bassi nel dominio salute generale $(48,05$ vs 49,96, $p=0,047)$. Analizzando invece il THYCA-QoL, il gruppo TTx+RAI mostrava una maggiore sensazione di freddo $(p=0,023)$. Il questionario FoP non mostrava invece differenze significative fra i due gruppi. Nessuna differenza fra i due gruppi veniva evidenziata dividendoli per rhTSH $v s$ ipotiroidismo e considerando l'attività di ${ }^{131}$ I somministrata.

In base a questi dati, gli autori concludono che il trattamento RAI potrebbe avere effetti negativi sulla QoL; pertanto, a fronte dello scarso beneficio clinico e dei possibili eventi avversi, soprattutto nei pazienti a basso rischio di recidiva, occorre tener conto anche del possibile impatto sulla QoL nel decidere se trattare o meno il paziente con RAI.

I limiti dello studio sono rappresentati dal fatto che il questionario è stato ottenuto a un solo punto del follow-up, dalla mancata analisi di possibili fattori confondenti e dal numero limitato di pazienti analizzati. Inoltre, occorre ricordare che la $\mathrm{QoL}$ in un paziente oncologico è inficiata da diversi fattori $[2,3]$, molti dei quali non vengono analizzati in questo studio. Tuttavia, il fatto che questi dati siano presenti dopo 4 anni circa dalla terapia iniziale e dopo che l'eutiroidismo sia stato mantenuto per molto tempo suggerisce che la decisione di procedere a un trattamento RAI dovrebbe essere considerata in maniera molto attenta e bilanciando benefici e rischi, includendo tra questi ultimi anche il possibile impatto negativo sulla QoL.

Funding Note Open access funding provided by Università di Pisa within the CRUI-CARE Agreement.

Nota della casa editrice Springer Nature rimane neutrale in riguardo alle rivendicazioni giurisdizionali nelle mappe pubblicate e nelle affiliazioni istituzionali.

Open Access This article is licensed under a Creative Commons Attribution 4.0 International License, which permits use, sharing, adaptation, distribution and reproduction in any medium or format, as long as you give appropriate credit to the original author(s) and the source, provide a link to the Creative Commons licence, and indicate if changes were made. The images or other third party material in this article are included in the article's Creative Commons licence, unless indicated otherwise in a credit line to the material. If material is not included in the article's Creative Commons licence and your intended use is not permitted by statutory regulation or exceeds the permitted use, you will need to obtain permission directly from the copyright holder. To view a copy of this licence, visit http://creativecommons.org/licenses/by/4.0/.

\section{Bibliografia}

1. Haugen BR, Alexander EK, Bible KC et al (2016) 2015 American Thyroid Association management guidelines for adult patients with thyroid nodules and differentiated thyroid cancer: the American Thyroid Association Guidelines Task Force on Thyroid Nodules and Differentiated Thyroid Cancer. Thyroid 26(1):1-133

2. Goldfarb M, Casillas J (2016) Thyroid cancer-specific quality of life and health-related quality of life in young adult thyroid cancer survivors. Thyroid 26(7):923-932

3. Husson O, Haak HR, Buffart LM et al (2013) Health-related quality of life and disease specific symptoms in long-term thyroid cancer survivors: a study from the population-based PROFILES registry. Acta Oncol 52(2):249-258 